\title{
Automatic Task Matching and Negotiated Vehicle Scheduling for Intelligent Logistics System
}

\author{
Xuanxuan Zhang \\ College of Economics and Business Administration, Chongqing University, Chongqing, CHINA
}

\section{SUMMARY}

The decision-making of logistics vehicle scheduling is difficult under varying constraints, multiple disturbances and strong time-variation. The multi-agent system (MAS) is a new approach to investigate the real-time decision-making of logistics vehicle scheduling. It satisfies the various requirements of the logistics system, such as the geographical distribution of vehicles, the dynamic changes of information, and the constant changes in consumer orders. In view of the theoretical and practical significance of the MAS, this paper explores the decision-making of logistics vehicle scheduling based on the MAS, and relies on two-level planning modelling method to construct the mathematical model of outsourcing-based container port vehicle scheduling problem. Then, an effectively exchange neighbourhood tabu search algorithm was designed to solve the model. Through the research, it is concluded that the proposed hierarchical decomposition method of logistics distribution task can reduce the overall scheduling difficulty and reduce the actual planning error effectively; the established MAS-based intelligent logistics scheduling model can minimize the total distribution cost through continuous adjustment of resources according to the distribution task. Finally, the feasibility of the proposed algorithm was verified by the results of a calculation example.

KEY WORDS: Intelligent logistics system; vehicle scheduling; automatic task matching; intelligent decision-making.

\section{INTRODUCTION}

The boom of global trade and e-commerce has propelled the diversification, commercialization, and transformation of logistics distribution [1,2]. Logistics, the liaison between producer and client, is affected by multiple factors, such as market environment and customer demand. Nowadays, the traditional point-to-point distribution model can no longer satisfy the demand in an increasingly competitive market [3]. To solve the problem, it is an imperative to set up an intelligent logistics system that highlights the importance of vehicle scheduling, considers the links between the upstream, midstream and downstream of the logistics chain and supports intelligent decision-making in logistics scheduling [4, 5]. 
The existing studies on logistics scheduling optimization mainly concentrate on the vehicle scheduling and routing [6-9] and exist as combinatorial scheduling optimization based on models, methods and algorithms. For model-based optimization, the actual logistics model is sometimes placed into a class of graph theory problems [10-14]; for method-based optimization, the actual logistics scheduling is solved as several basic problems, and then combined into the original problem [15, 16]; for algorithm-based optimization, the scheduling is improved by precise algorithms and heuristic algorithms, such as probability analysis, shortest path method, coverage algorithm, interactive optimization method, branch and bound method, etc. [17-20].

One of the most popular algorithms for logistics scheduling optimization is a dynamic approach based on multi-agent system (MAS). The MAS, a collection of multiple intelligent agents, aims to transform large and complex systems into small, easy-to-manage systems that communicate and coordinate with each other. This approach is good at describing dynamic complex systems, thanks to its natural attributes like collaboration, parallelism, robustness, scalability and distributed solutions. As a result, the MAS has often been used as a theoretical reference for building complex dynamic systems and played an increasingly important role in the intelligent decision support system. Thanks to its intelligence, adaptability and autonomy, the MAS outperforms other algorithms in the research of intelligent logistics systems [21-24]. For instance, the MAS has been preliminarily adopted for the optimal allocation of job-shop resources $[25,26]$, the planning of distributed process flow $[27,28]$ and the dynamic scheduling of the production system $[29,30]$. However, there is still no report on its application in logistics system for automatic task matching and negotiated scheduling of distribution vehicles.

To fill the gap, this paper attempts to improve the traditional logistics scheduling with the MAS. Firstly, an automatic task matching and negotiated scheduling method was proposed based on the intelligent logistics system. Then, the Agent framework model was suggested to suite the method. Finally, the proposed method was verified through a case study.

\section{VEHICLE SCHEDULING MODEL BASED ON INTELLIGENT LOGISTICS SYSTEM}

\subsection{LOGISTICS SCHEDULING FOR GENERAL OPERATIONS}

As shown in Figure 1, in the traditional logistics scheduling process, the products leave from the upstream and pass through the delivery centre before finally reaching the client.

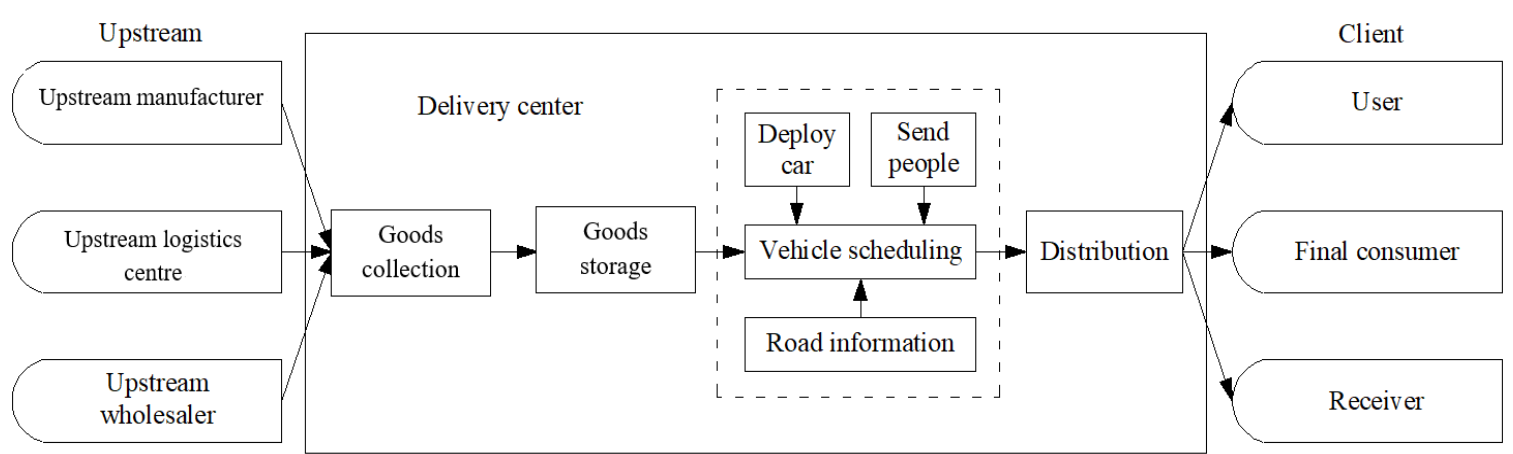

Fig. 1 Traditional logistics scheduling process 
Logistics scheduling, the core of the entire logistics process, consists of cargo distribution, vehicle scheduling and routing. The distribution of goods can be abstracted into the following knapsack problem:

$$
\max f(X)=\sum_{j=1}^{N} p_{j} x_{j} \text { s.t. }\left\{\begin{array}{l}
\sum_{j=1}^{N} w_{j} x_{j} \leq W \\
x_{j} \in\{0,1\},(j=1,2, \ldots, N)
\end{array}\right.
$$

The optimization goal is to maximize the value $f(X)$ of the cargo combination $X$ of each vehicle. The vehicle scheduling problem can be solved with the following mathematical model of the assignment problem:

$$
\min f=\sum_{i=1}^{n} \sum_{j=1}^{n} c_{i j} x_{i j} \quad \text { s.t. }\left\{\begin{array}{l}
\sum_{i=1}^{n} x_{i j}=1, j=1,2, \ldots, n \\
\sum_{j=1}^{n} x_{i j}=1, i=1,2, \ldots, n \\
x_{i j}=\operatorname{lor} 1, i, j=1,2, \ldots, n
\end{array}\right.
$$

where $\min f$ is the minimum total cost of vehicle scheduling. The routing can be simplified as the solution to the traveling salesman problem (TSP):

$$
\min f=\sum_{i \neq j} c_{i j} x_{i j} \quad \text { s.t. }\left\{\begin{array}{l}
\sum_{j \neq i} x_{i j}=1, i \in V \\
\sum_{i \neq j} x_{i j}=1, j \in V \\
\sum_{i, j \in V} x_{i j} \leq|K|-1, K \subset V \\
x_{i j}=\text { oor } 1, i, j \in V
\end{array}\right.
$$

According to the above formulas, the overall logistics flow can be expressed as:

$$
\operatorname{minz}=\sum_{i} \sum_{j} \sum_{k} c_{i j} x_{i j k} \quad \text { s.t. }\left\{\begin{array}{l}
\sum_{i} g_{i} y_{k i} \leq q ; \forall k \\
\sum_{k} y_{k i}=1, i=1,2, \ldots, l \\
\sum_{i} x_{i j k}=y_{k i}, j=1,2, \ldots, l ; \forall k \\
\sum_{j} x_{i j k}=y_{k i}, i=1,2, \ldots, l ; \forall k \\
X=\left(x_{i j k}\right) \in D \\
x_{i j k}=\text { oor } 1, i, j=0,1,2, \ldots, l ; \forall k \\
y_{k i}=0 \text { or } 1, i=0,1,2, \ldots, l ; \forall k \\
t_{0 i k} \in\left[E T_{i}, L T_{i}\right], \forall y_{k i}=1
\end{array}\right.
$$

where $t_{0 i k}$ is the delivery time of the goods; $c_{i j}$ is the distribution cost of a vehicle from distribution site $i$ to distribution site $j$ (including the vehicle cost, the personnel cost, etc.); $x_{i j k}$ is the correlation coefficient of the route $\left(x_{i j k}=1\right.$ if vehicle $k$ drives from distribution site $i$ to distribution site $j$ and $x_{i j k}=0$ if otherwise). An optimization function that minimizes the number of vehicles or the length of the route can be derived from Eq. (4). 


\subsection{MAS-BASED LOGISTICS SCHEDULING MODEL}

Here, the MAS is introduced to improve the traditional logistics scheduling, forming a MASbased model. The core processing structure is the multi-agent planning system in Figure 2.

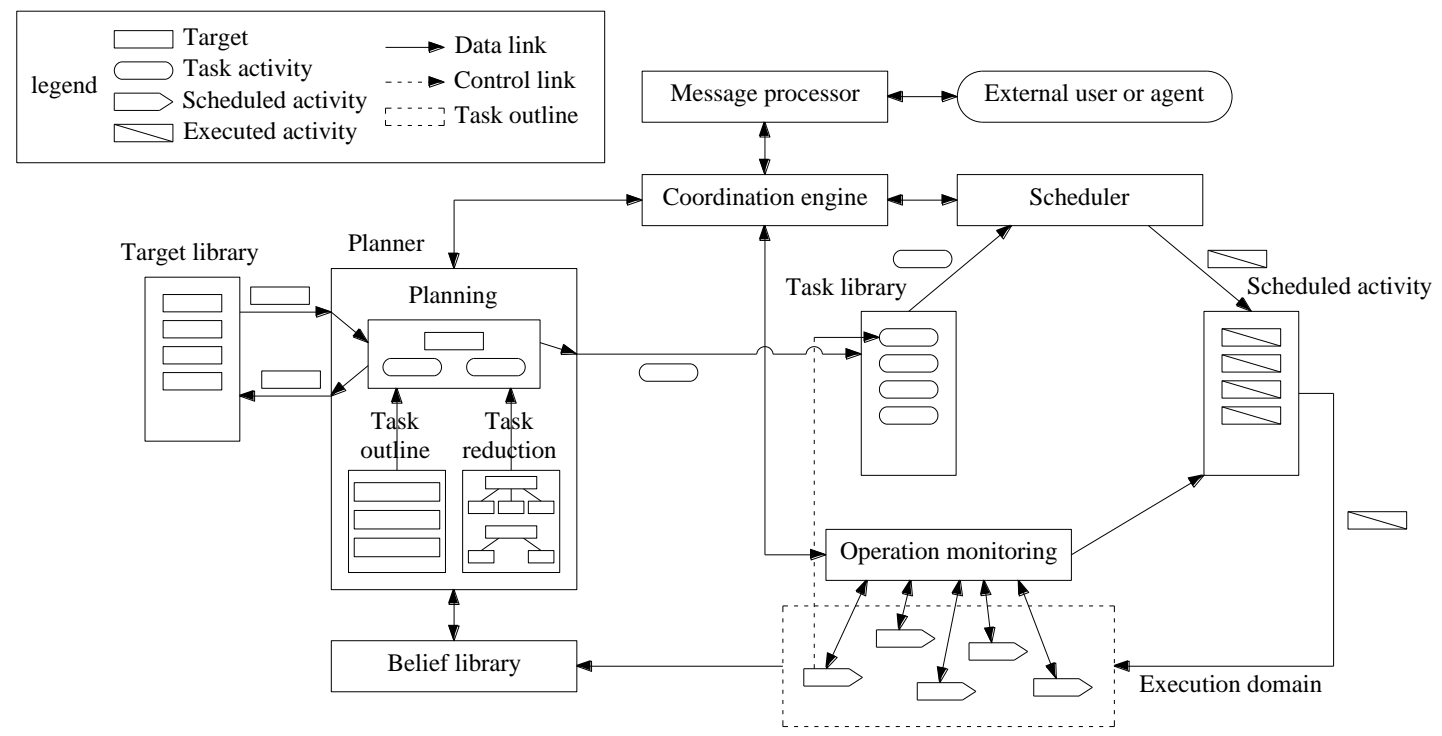

Fig. 2 Multi-agent planning system

The system mainly consists of a target library, a task library and an information library. Each library contains agents with different functions. The MAS-based logistics distribution process can be simplified to the multi-agent model in Figure 3 below.

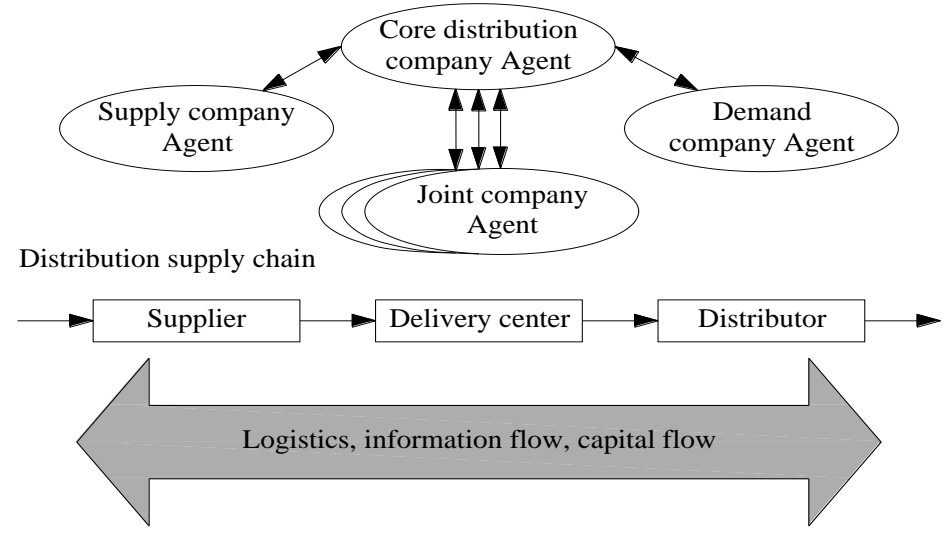

Fig. 3 Multi-agent model for the MAS-based logistics distribution

On this basis, the author established a multi-agent vehicle scheduling management system model for intelligent logistics (Figure 4). The above-mentioned multi-agent planning system is placed at the centre of this system model. In addition, there is a cargo distribution agent, a vehicle agent, and a route information agent in this model. Each agent has its independent reasoning and communication capabilities. The agents communicate in messages coded in XML. 


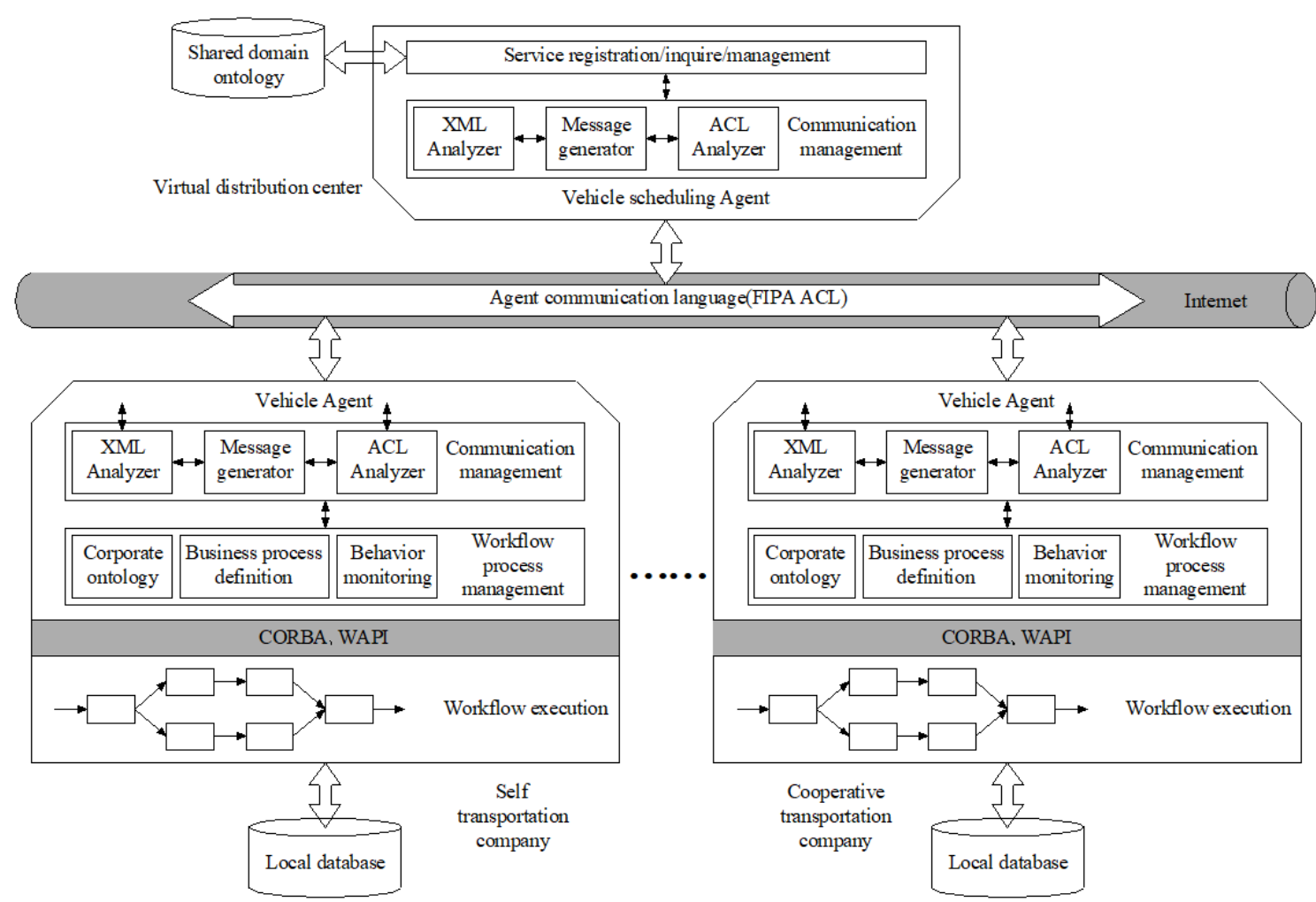

Fig. 4 Multi-agent vehicle dispatch management system for intelligent logistics

Once the system model receives a batch of orders, the planning system agent will send the order delivery information to other execution agents. Then, the XML parser in each agent will encode and parse the information. The parsed commands will be transmitted via the ACL to the agents to initiate relevant operations.

\section{VEHICLE SCHEDULING METHOD FOR INTELLIGENT LOGISTICS SYSTEM}

\subsection{LOGISTICS SCHEDULING FOR GENERAL OPERATIONS}

Through further analysis, a vehicle scheduling method was put forward for intelligent logistics system and the overall workflow of the delivery centre was clarified (Figure 5).

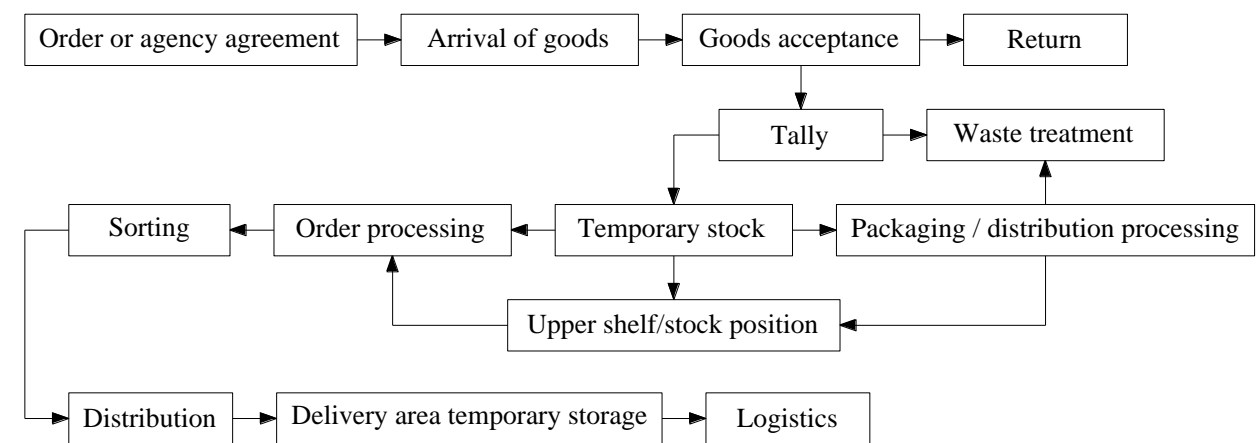

Fig. 5 Overall workflow of the distribution centre 
Considering the huge order volume of the delivery centre, the distribution items should be decomposed as follows: simplify different items as several units $X$, and decompose each unit into subunits $X_{1} \sim X_{n}$ that satisfy:

$$
\begin{gathered}
X=X_{1} \cup X_{2} \cup \ldots \cup X_{i} \cup \ldots \cup X_{n} \\
X_{i} \cap X_{j}=\Phi(i \neq j) \\
C_{X}=\sum C_{X_{1}}
\end{gathered}
$$

The subunits should have the same attributes, be independent, integrable and time-varying, and be able to distinguish between different job contents and responsible persons.

The distribution task decomposition of intelligent logistics system is shown in Figure 6. The overall distribution task can be decomposed into several subunits according to the abovementioned MAS-based logistics scheduling model. The entire distribution task can be reconstructed from the smallest decomposable units, marked as $P_{e}$.

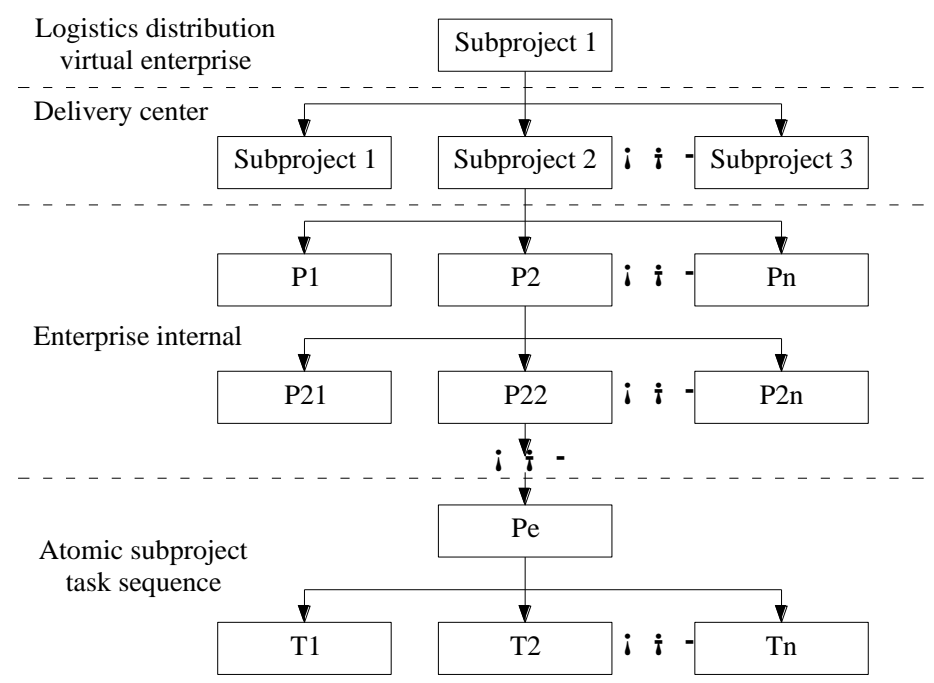

Fig. 6 Distribution task decomposition of intelligent logistics system

The primary subunits include the collection of goods, storage of goods, vehicle scheduling and delivery of goods. Among them, the collection of goods can be further divided into two secondary subunits: order acceptance and order issuance/charging; the storage of goods can be further divided into goods acceptance, processing, and goods placement; vehicle dispatching can be further divided into goods assembling, route planning and fuel management; the delivery of goods can be further divided into inspection of goods, issuance of orders and shipment of goods. 




Fig. 7 Resource planning for distribution scheduling of intelligent logistics system

As shown in Figure 7, the resource planning for distribution scheduling of intelligent logistics system can minimize the total distribution cost through continuous adjustment of the resource allocation according to the distribution task.

\subsection{IMPROVED EXCHANGE NEIGHBOURHOOD TABU SEARCH ALGORITHM}

The improved exchange neighbourhood tabu search algorithm was adopted to minimize the total cost of logistics distribution. Assuming that each delivery centre has $k$ vehicles and several orders, the initial solution of the job sequence corresponding to the $k$ vehicles was generated by the insertion method, while the remaining orders were outsourced. In this way, a total of $k+1$ task sequences were generated.

Under the exchange structure of the adopted algorithm, two task sequences were selected to exchange their orders, forming new task sequences. Since the value of $\lambda$ in the algorithm is either 1 or 2 , eight exchange operators (e.g. $(0,1),(1,1)$ and $(2,1))$ can be generated. Note that $(1,1)$ means one order is exchanged into another task sequence. The computation should be terminated when the iteration results reach the pre-set threshold or the maximum number of iterations.

The specific steps of the adopted algorithm are as follows:

(a) Randomly generate the initial solution $S$, denote the best-known solution as $S_{b}$, and make it the initial solution, i.e. $S=S_{b}$;

(b) Initialize various parameters in the algorithm;

(c) Perform a neighbourhood search on $S$, and update the completed and to-be-completed task sequences in real time;

(d) The objective function of the optimization can be expressed as: 


$$
\begin{gathered}
\text { PHASE I: Minimize } \sum_{i=1}^{N} P_{i}\left(\sum_{k=1}^{K} \sum_{m=1}^{M} X_{i k m}\right) \\
\text { PHASE II: Minimize } \sum_{i=k+1}^{N} \sum_{l=1}^{L} S_{l i} X_{l i} \\
\text { PHASE III: Minimize } \sum_{i=1}^{N} P_{i}\left(\sum_{k=1}^{K} \sum_{m=1}^{M} X_{i k m}\right)+\sum_{i=1}^{N} \sum_{l=1}^{L} S_{l i} X_{l i}
\end{gathered}
$$

Eqs. (8) to (10) respectively specify the minimum total distribution cost of the orders delivered by the vehicles of the delivery centres, the minimum total distribution cost of the orders delivered by outsourced vehicles, and the minimum total distribution cost of the orders delivered by vehicles of the delivery centres and outsourced vehicles. The optimal plan obtained by Eqs. (8) to (10) is marked as $S S$. If $\operatorname{Cost}\left(S_{b}\right)>\operatorname{Cost}(S S)$, update $S$ into $S S$ and start the iteration and computation again:

$$
\text { If }\left\{\operatorname{Cost}\left(S_{b}\right)>\operatorname{Cost}(S)\right\} \quad S_{b}=S, m=m+1
$$

Otherwise, use the original value for the computation.

\section{CASE STUDY}

The logistics scheduling of a large port was selected to validate of the proposed automatic task matching and negotiated scheduling method for intelligent logistics system. There are three types of vehicle scheduling for the logistics distribution at the port (Figure 8).

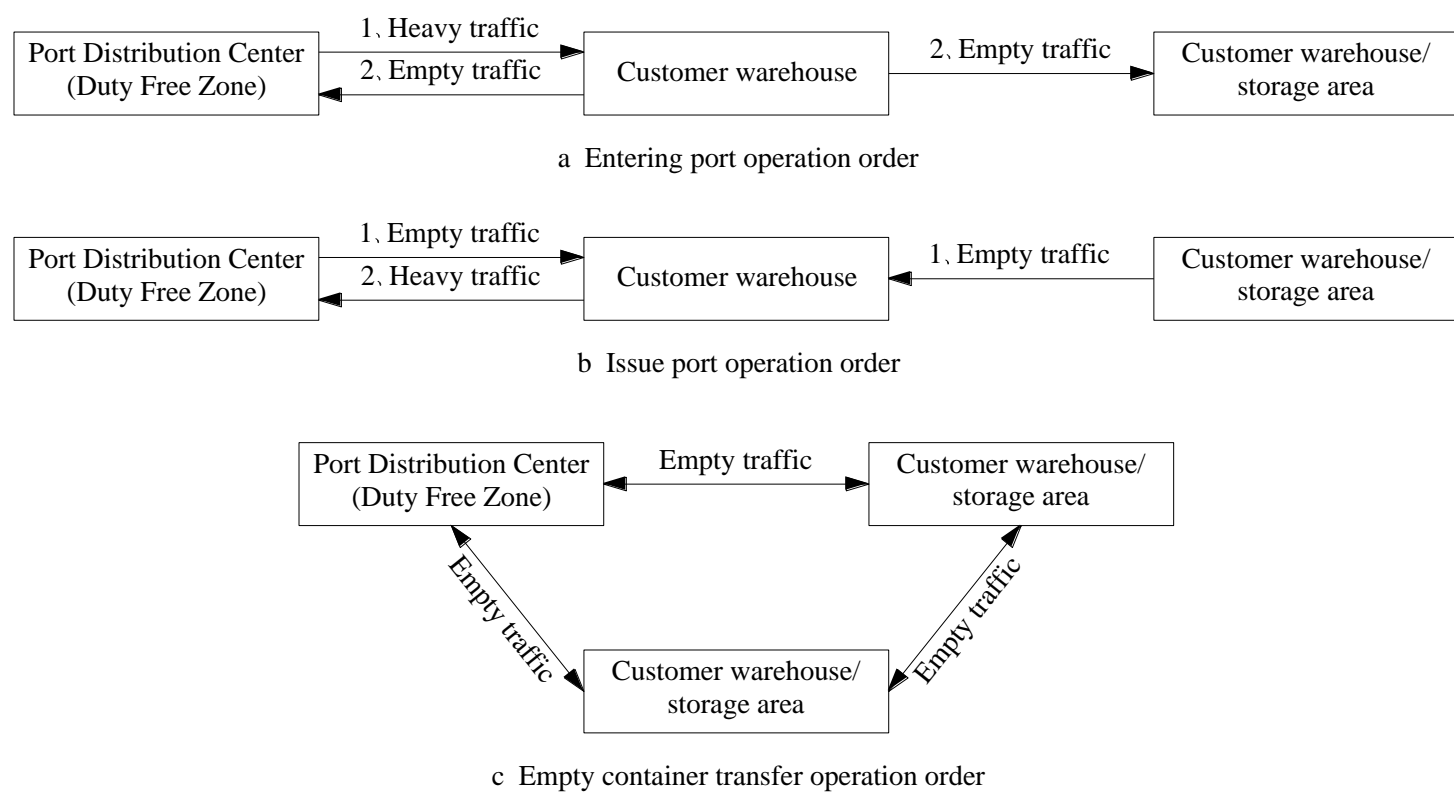

Fig. 8 Types of vehicle scheduling for the logistics distribution at the port

In light of our approach, a port vehicle scheduling agent model was created for intelligent logistics system (Figure 9). The model involves the task announcement, task assignment, agent design (environment agent, communication agent, skill agent, identification agent), and route planning. 


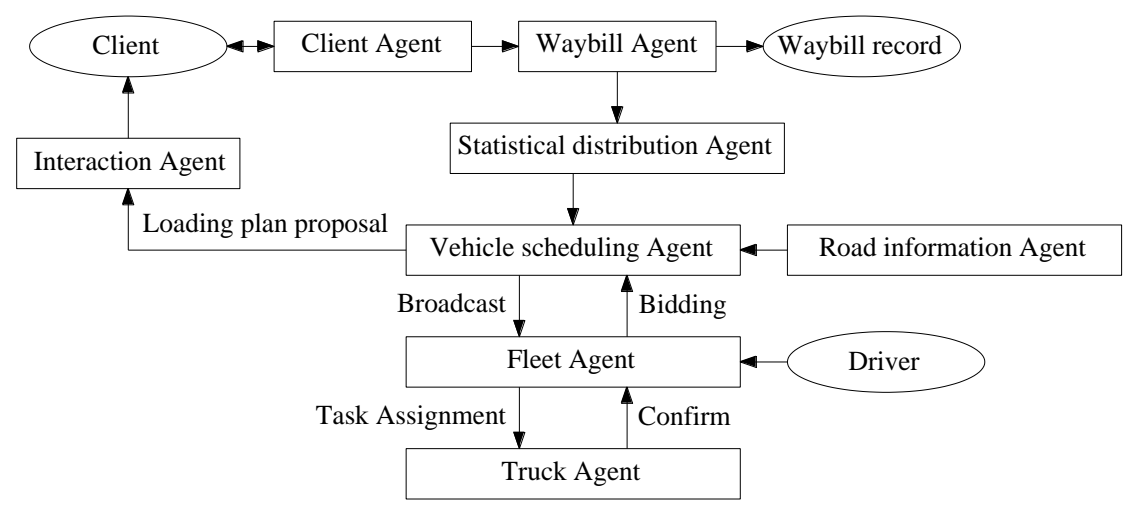

Fig. 9 Port vehicle scheduling agent model for intelligent logistics system

The orders at the port were processed according to Figure 10, where $H_{i 1}$ and $H_{i 2}$ are the processing time of the logistics operation at the start point and the end point, respectively; $W_{m k 1}$ and $W_{m k 2}$ are the waiting time of the logistics operation at the start point and the end point, respectively; $D_{i}$ is the distribution form; $R_{i 0} \sim R_{i 1}$ and $R_{i 2} \sim R_{i 3}$ are the longest allowable operation time.

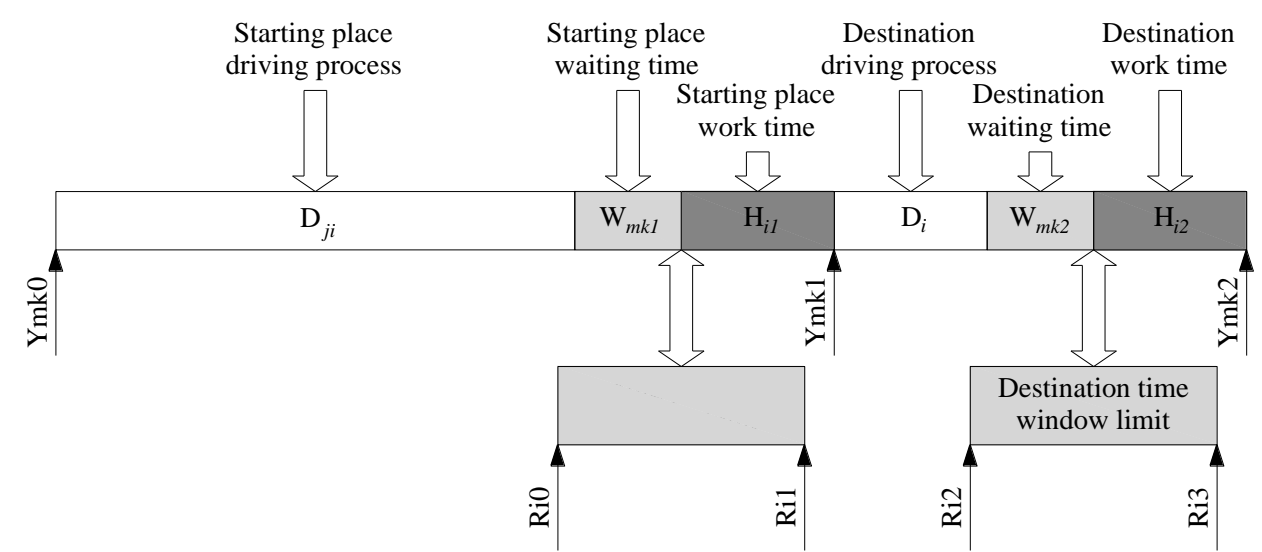

Fig. 10 Workflow of order processing at the port

Next, the logistics distribution of the container goods at the port was computed by the proposed model and relevant algorithms. The relationship between the number of iterations and the relative total cost is presented in Figure 11.

The relative total cost can be expressed as:

$$
\min \sum_{i=1}^{N} P_{i}\left(\sum_{k=1}^{K} \sum_{m=1}^{M} X_{i k m}\right)
$$

where $N$ is the number of orders; $K$ is the number of distribution vehicles; $M$ is the maximum number of orders that can be processed each day by each vehicle.

Figure 11 shows that the proposed model handled the port orders excellently. The total distribution cost plunged with the growing number of iterations, and reached the optimal scheduling state after the $16^{\text {th }}$ iterations. In addition, the proposed algorithm has never converged to the local optimum, indicating that it can effectively prevent premature convergence. Thus, it is feasible to use the proposed algorithm for the intelligent control of logistics distribution. 


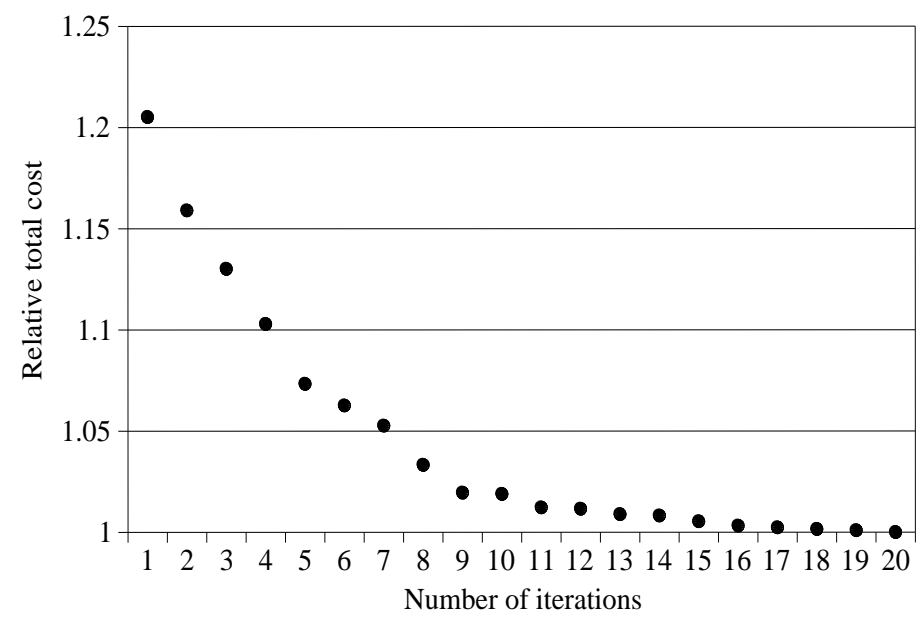

Fig. 11 The relationship between the number of iterations and the relative total cost

Next, the proposed algorithm was further contrasted with the traditional insertion method and neighbourhood search algorithm against the same logistics plan. The comparison in Figure 12 shows that the insertion method brought the highest relative total cost (mean value: 1.22), followed by the neighbourhood search algorithm (mean value: 1.14). The proposed algorithm achieved a much lower distribution cost than the two contrastive algorithms.

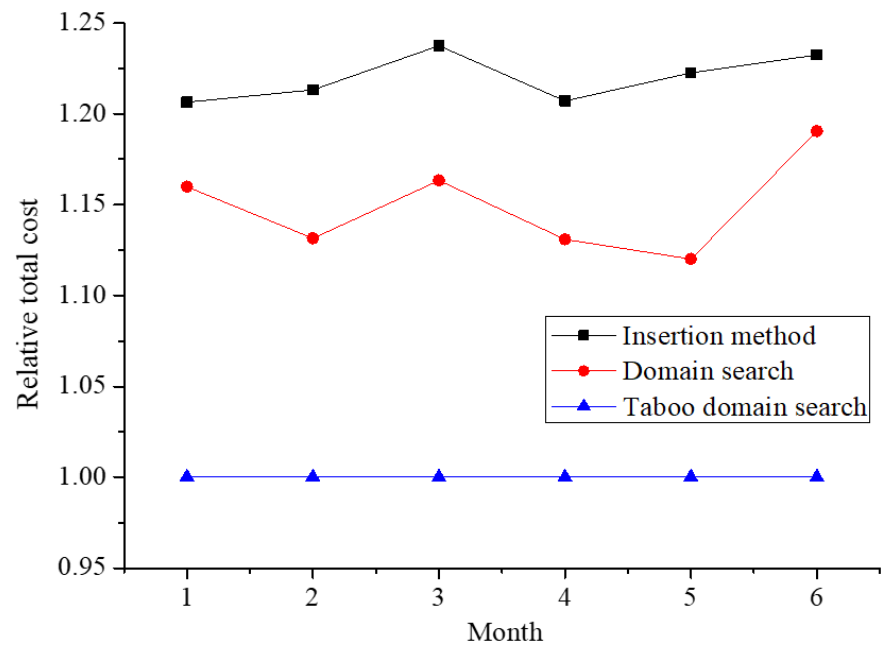

Fig. 12 The relative total costs of the three algorithms

\section{CONCLUSIONS}

This paper introduces the innovative technology of the MAS to improve the traditional logistics scheduling, proposes an automatic task matching and negotiated scheduling method for intelligent logistics system, and constructs a corresponding agent framework model. The research conclusions are as follows:

(1) The multi-layer decomposition of the overall logistics scheduling task greatly reduces the scheduling difficulty of the overall logistics process, and, to some extent, prevents the computing error caused by incomplete data and uncertain indices. 
(2) The MAS-based intelligent system logistics scheduling model supports automatic task matching and auto-negotiation. During distribution, the total distribution cost can be minimized through continuous adjustment according to the delivery task.

(3) The case study demonstrates the effectiveness and superiority of the proposed method. Compared with the insertion method and the neighbourhood search algorithm, the proposed method can effectively avoid premature convergence.

\section{REFERENCES}

[1] C. Bo, Logistics scheduling with batching and transportation, European Journal of Operational Research, Vol. 189, pp. 871-876, 2008. http://dx.doi.org/10.1002/nav.20078

[2] L. Jiang, H.Y. Wang, Research on workstation-oriented production logistics optimization of Chinese discrete manufacturing enterprises, Advanced Materials Research, pp. 17511757, 2014. http://dx.doi.org/10.4028/www.scientific.net/amr.834-836.1751

[3] H.S. Hwang, Design of supply-chain logistics system considering service level. Computers \& Industrial Engineering, Vol. 43, pp. 283-297, 2002. http://dx.doi.org/10.1016/s0360-8352(02)00075-x

[4] L.X. Zhou, K. Liu, Intelligent logistics transportation system, Journal of Tongji University, Vol. 58, No. 58, pp. 55-70, 2002. http://dx.doi.org/10.1108/17410400910921083

[5] A. Adamski, Hierarchical integrated intelligent logistics system platform, Procedia Social and Behavioural Sciences, Vol. 20, No. 6, pp. 1004-1016, 2011. http://dx.doi.org/10.1016/j.sbspro.2011.08.109

[6] L. Bodin, B. Golden, B. Classification in vehicle routing and scheduling, Networks, Vol. 11, No. 2, pp. 97-108, 2010. http://dx.doi.org/10.1002/net.3230110204

[7] B. Funke, T. Grünert, S. Irnich, Local search for vehicle routing and scheduling problems: review and conceptual integration, Journal of Heuristics, Vol. 11, No. 4, pp. 267-306, 2005. http://dx.doi.org/10.1007/s10732-005-1997-2

[8] H. Wang, C.Y. Lee, Production and transport logistics scheduling with two transport mode choices, Naval Research Logistics, Vol. 52, No. 8, pp. 796-809, 2010.

http://dx.doi.org/10.1002/nav.20116

[9] J. Barceló, H. Grzybowska, S. Pardo, Vehicle routing and scheduling models, simulation and city logistics, Operations Research/computer Science Interfaces, Vol. 38, pp. 163-195, 2007. http://dx.doi.org/10.1007/978-0-387-71722-7_8

[10] P. Carraresi, G. Gallo, Network models for vehicle and crew scheduling, European Journal of Operational Research, Vol. 16, No. 2, pp. 139-151, 2007. http://dx.doi.org/10.1016/0377-2217(84)90068-7

[11] C.M. Meyer, H. Kopfer, A.L. Kok, M. Schutten, Distributed decision making in combined vehicle routing and break scheduling, Vehicle Routing Under Consideration of Driving \& Working Hours, pp. 125-133, 2009. http://dx.doi.org/10.1007/978-3-642-11996-5_12

[12] M. Lai, H. Yang, S. Yang, J. Zhao, Y. Xu, Cyber-physical logistics system-based vehicle routing optimization, Journal of Industrial \& Management Optimization, Vol. 10, No. 3, pp. 701-715, 2017. http://dx.doi.org/10.3934/jimo.2014.10.701 
[13] X. Qi, A logistics scheduling model: inventory cost reduction by batching, Naval Research Logistics, Vol. 52, No. 4, pp. 312-320, 2006. http://dx.doi.org/10.1002/nav.20078

[14] X.T. Qi, A logistics scheduling model: scheduling and transhipment for two processing centers, Iie Transactions, Vol. 38, No. 7, pp. 537-546, 2006.

http://dx.doi.org/10.1080/074081791009022

[15] B.Z. Li, Y.Q. Zhou, S.H. Lan, J.G. Yang, The information modelling and intelligent optimization method for logistics vehicle routing and scheduling with multi-objective and multi-constraint, Journal of Donghua University (English Edition), Vol. 24, No. 4, pp. 455459, 2007.

[16] B. Vahdani, R. Tavakkoli-Moghaddam, M. Zandieh, J. Razmi, Vehicle routing scheduling using an enhanced hybrid optimization approach, Journal of Intelligent Manufacturing, Vol. 23, No. 3, pp. 759-774, 2012. http://dx.doi.org/10.1007/s10845-010-0427-y

[17] M. Boudia, M.A. Ouldlouly, C. Prins, Fast heuristics for a combined production planning and vehicle routing problem, Production Planning \& Control, Vol. 19, No. 2, pp. 85-96, 2008. http://dx.doi.org/10.1080/09537280801893356

[18] A.M. Campbell, M. Savelsbergh, Efficient insertion heuristics for vehicle routing and scheduling problems, Transportation Science, Vol. 38, No. 3, pp. 369-378, 2004. http://dx.doi.org/10.1287/trsc.1030.0046

[19] T. Miyamoto, N. Tsujimoto, S. Kumagai, A cooperative algorithm for autonomous distributed vehicle systems with finite buffer capacity, Ieice Transactions on Fundamentals of Electronics Communications \& Computer Sciences, Vol. E88-A, No. 11, pp. 3036-3044, 2005. http://dx.doi.org/10.1093/ietfec/e88-a.11.3036

[20] F.S. Chang, J.S. Wu, C.N. Lee, H.C. Shen, Greedy-search-based multi-objective genetic algorithm for emergency logistics scheduling, Expert Systems with Applications, Vol. 41, No. 6, pp. 2947-2956, 2014. http://dx.doi.org/10.1016/j.eswa.2013.10.026

[21] Y. Chen, X. Wang, F. Lin, K. Tang, The multi-agent modelling of production logistics system and the shop dynamic scheduling optimization in discrete manufacturing enterprises, Physiology \& Behaviour, Vol. 63, No. 3, pp. 604-610, 2006.

http://dx.doi.org/10.1049/cp:20060833

[22] E. Taniguchi, T. Yamada, M. Okamoto, Multi-agent modelling for evaluating dynamic vehicle routing and scheduling systems, Proceedings of the Eastern Asia Society for Transportation Studies, pp. 134-134, 2007.

[23] M. Balmer, K. Nagel, B. Raney, Large-scale multi-agent simulations for transportation applications, Journal of Intelligent Transportation Systems, Vol. 8, No. 4, pp. 205-221, 2004. http://dx.doi.org/10.1080/15472450490523892

[24] A. Kouider, B. Bouzouia, Multi-agent job shop scheduling system based on co-operative approach of idle time minimization, International Journal of Production Research, Vol. 50, No. 2, pp. 409-424, 2012. http://dx.doi.org/10.1080/00207543.2010.539276

[25] N.O. Amelina, A.N. Lada, I.V. Mayorov, P.O. Skobelev, A.V. Tsarev, Cargo transportation models analysis using multi-agent adaptive real-time truck scheduling system, Institute of Control Sciences of Complex Systems, pp. 31-37, 2013. http://dx.doi.org/10.5220/0004225502440249 
[26] M.E. Aydin, T.C. Fogarty, A simulated annealing algorithm for multi-agent systems: a jobshop scheduling application, Journal of Intelligent Manufacturing, Vol. 15, No. 6, pp. 805814, 2004. http://dx.doi.org/10.1023/b:jims.0000042665.10086.cf

[27] S.M. Ghamrawy, A.I. Desouky, M. Saleh, Implementing a decision support module in distributed multi-agent system for task allocation using granular rough model, International Journal of Engineering and Technology, Vol. 3, No. 1, pp. 85-95, 2011. http://dx.doi.org/10.7763/ijet.2011.v3.205

[28] P. Renna, Deteriorating job scheduling problem in a job-shop manufacturing system by multi-agent system, International Journal of Computer Integrated Manufacturing, Vol. 28, No. 9, 936-945, 2015. http://dx.doi.org/10.1080/0951192x.2014.928747

[29] P. Skobelev, Multi-agent systems for real time resource allocation, scheduling, optimization and controlling: industrial applications, Communication- and ComputationBounded Agents in Multi-agent Simulations, Vol. 6867, pp. 1-14, 2014. http://dx.doi.org/10.1007/978-3-642-23181-0_1

[30] W. Xiong, D. Fu, A new immune multi-agent system for the flexible job shop scheduling problem, Journal of Intelligent Manufacturing, No. 6, pp. 1-17, 2015.

http://dx.doi.org/10.1007/s10845-015-1137-2 